\title{
Opportunistic Relay Selection with Outdated CSI: Outage Probability and Diversity Analysis
}

\author{
Jose Lopez Vicario, Member, IEEE, Albert Bel, Jose A. Lopez-Salcedo, Member, IEEE, \\ and Gonzalo Seco, Senior Member, IEEE
}

\begin{abstract}
In this paper, we analyze the outage probability and diversity order of opportunistic relay selection in a scenario based on decode and forward and where the available channel state information (CSI) is outdated. The study is conducted analytically by obtaining a closed-form expression for the outage probability, which is defined as the probability that the instantaneous capacity is below a target value. We derive high-SNR approximations for the outage probability. By doing so, we demonstrate that the diversity order of the system is reduced to 1 when CSI is outdated, being this behavior independent of the level of CSI accuracy. A physical explanation for this extreme loss of diversity is provided along with numerical results to support the analytical study.
\end{abstract}

Index Terms-Cooperative communications, opportunistic relay selection, outdated CSI, diversity gain.

\section{INTRODUCTION}

C OOPERATIVE diversity has been shown to be an efficient way to combat wireless impairments using low complexity terminals [1]-[3]. Basically, these schemes allow for the exploitation of spatial diversity gains without the need of multi-antenna technology. Different spatial paths are provided by sending/receiving the information to/from a set of cooperating terminals working as relays. By doing so, most of the advantages of multiple-input multiple-output (MIMO) techniques [4] can be extracted while keeping the complexity of the individual terminals reduced.

Among the set of cooperative techniques, opportunistic relay selection (ORS) is a useful strategy for practical implementation [5], [6]. This is because ORS is a low complexity strategy consisting only in activating the best relay (in accordance to a given performance metric). Apart from the inherent simplicity of the proposed technique, this strategy avoids the need of synchronization (needed by most distributed spacetime coding schemes) and reduces the power consumption of the terminals.

When ORS is implemented in a real system, however, there may exist a delay between the instant when the relay selection process is encompassed and the actual transmission of data from the selected relay. In other words, the channel state of the selected relay at the selection decision instant can substantially differ from the actual channel during data transmission and, as a result, system performance is affected.

Manuscript received July 11, 2008; revised November 17, 2008; accepted February 27, 2009. The associate editor coordinating the review of this letter and approving it for publication was Y.-C. Ko.

The authors are with the Universitat Autonoma de Barcelona (UAB), 08193 Bellaterra, Spain (e-mail: vicario@ieee.org; \{albert.bel, jose.salcedo, gonzalo.seco\}@uab.es).

This work was supported by the Spanish Government Project TEC200806305/TEC.

Digital Object Identifier 10.1109/TWC.2009.081561
The study of the impact of outdated channel state information (CSI) on ORS has been addressed in some works. For instance, it was shown in [7] that a selection relaying mechanism based on localization knowledge can outperform an opportunistic scheme with instantaneous information. The reason for that was that the system may work better when decisions are made based on location information instead of instantaneous but outdated CSI. The viability of using a cooperative scheme in WiMAX networks was addressed in [8], showing that ORS-based cooperation causes a performance loss when the available CSI is outdated and the number of relays is low.

In this paper, we concentrate our efforts on the analytical study of the behavior of ORS when CSI is outdated and the relaying strategy is based on decode and forward. To do so, we derive the exact expression for the outage probability. Since the provided expression is barely tractable, we also provide an approximation for the high-SNR regime. By doing so, we prove that the diversity order of the system is always equal to 1 when available CSI is not exact, regardless the degree of accuracy.

\section{System Model}

Consider a wireless network where one mobile unit (source) sends information to the base station (destination). In order to improve system performance, a cooperative mechanism is considered. In particular, an ORS strategy is adopted in a scenario with $K$ mobile units of the network working as relays. For the sake of notation simplicity, we define an arbitrary link $A$ - $B$ between two nodes $A$ and $B$. Node $A$ can be the source $(A=S)$ or the $k$-th relay $(A=k)$, while node $B$ can correspond to the $k$-th relay $(B=k)$ or to the destination $(B=D)$. With this model in mind, the received signal in the link $A-B$ can be written as follows:

$$
r_{B}=h_{A, B} x_{A}+n_{B}
$$

where $x_{A} \in \mathbb{C}$ is the transmitted symbol from node $A$ with power $P_{A}=\mathbb{E}\left[\left|x_{A}\right|^{2}\right], n_{B} \in \mathbb{C}$ is AWGN noise with zero mean and variance $\sigma_{n}^{2}$ (independent of the value of $B), h_{A, B} \in \mathbb{C}$ is the channel response between nodes $A$ and $B$ modeled as $h_{A, B} \sim \mathcal{C N}(0,1)$ (Rayleigh fading). For mathematical convenience, we assume a block-fading channel where the channel response remains constant during one time-slot and that the different channels (for changing A or B) are independent and identically distributed (i.i.d.). Concerning power allocation, we consider that total transmit power of the system, $P$, is evenly distributed among the source and the selected relay, $k^{*}$, i.e. $P_{S}=P_{k^{*}}=0.5 P$. We denote 


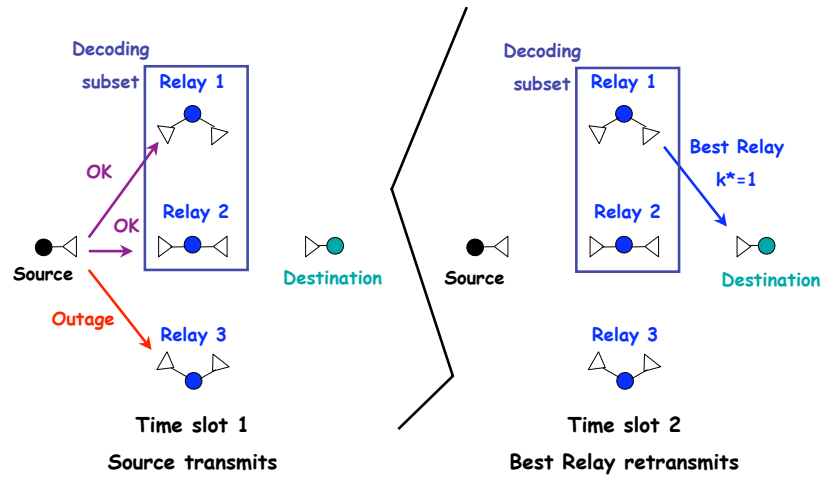

Fig. 1: Cooperative communications scheme based on ORS with DF.

by $\gamma_{A, B}=P_{A}\left|h_{A, B}\right|^{2} / \sigma_{n}^{2}$ the instantaneous signal-to-noise ratio (SNR) experienced in the link $A-B$ in a given timeslot and by $\bar{\gamma}_{A, B}=P_{A} / \sigma_{n}^{2}$ its long-term average. Thanks to the homogenous nature of the proposed system, we unify the notation in the sequel by denoting the average SNR per link as $\bar{\gamma}=0.5 P / \sigma_{n}^{2}$.

Concerning the relaying procedure, we consider a halfduplex two-hop decode and forward (DF) protocol as relaying strategy [3]. When using DF in an ORS scheme, only the best relay is allowed to cooperate with the source [6]. More specifically, the subset of relays able to decode the information is named as the decoding subset $\mathcal{D S}$ and, from that subset, the relay with the best relay-to-destination channel quality retransmits the information (see figure 1). In particular, the relay maximizing the SNR in the link $k-D$ is selected:

$$
k^{*}=\arg \max _{k \in \mathcal{D} \mathcal{S}}\left\{\hat{\gamma}_{k, D}\right\}
$$

where $\hat{\gamma}_{k, D}$ is the SNR available at the selection instant, which can differ from the actual SNR $\gamma_{k, D}$ during information retransmission due to channel variations ${ }^{1}$. Indeed, we assume that $\hat{\gamma}_{k, D}$ are obtained from $\hat{h}_{k, D}$ (i.e., $\hat{\gamma}_{k, D}=\left|\hat{h}_{k, D}\right|^{2} \bar{\gamma}$ ), which is an outdated version of $h_{k, D}$. Then, these two random variables are jointly Gaussian and, hence, $h_{k, D}$ conditioned on $\hat{h}_{k, D}$ follows a Gaussian distribution [9]:

$$
h_{k, D} \mid \hat{h}_{k, D} \sim \mathcal{C N}\left(\rho_{k} \hat{h}_{k, D}, 1-\rho_{k}^{2}\right)
$$

where parameter $\rho_{k}$ is the correlation coefficient between the envelopes of $h_{k, D}$ and $\hat{h}_{k, D}$, having different expressions according to the channel model ${ }^{2}$. For the sake of mathematical tractability, we assume in the sequel a scenario where parameter $\rho_{k}$ is the same for all the relays (i.e., $\rho_{k}=\rho \forall k$ ).

From the above discussion, it is straightforward to show that the actual SNR, $\gamma_{k, D}$, conditioned on its estimate, $\hat{\gamma}_{k, D}$, follows a non-central chi-square distribution with 2 degrees of freedom, whose probability density function (pdf) takes the

\footnotetext{
${ }^{1}$ Further details concerning the characteristics of the time delay between the instants when the selection process is encompassed and the actual transmission of data from the selected relay takes place can be found in [8].

${ }^{2}$ Under the assumption of a Jakes' model, for instance, the correlation coefficient takes the value $\rho_{k}=J_{o}\left(2 \pi f_{d_{k}} T_{D_{k}}\right)$, where $f_{d_{k}}$ stands for the Doppler frequency, $T_{D_{k}}$ is the delay between relay selection and information retransmission instants, and $J_{o}(\cdot)$ denotes the zero-order Bessel function of the first kind.
}

following expression [9]:

$$
\begin{aligned}
& f_{\gamma_{k, D} \mid \hat{\gamma}_{k, D}}\left(\gamma_{k, D} \mid \hat{\gamma}_{k, D}\right)=\frac{1}{\bar{\gamma}\left(1-\rho^{2}\right)} e^{\frac{-\left(\gamma_{k, D}+\rho^{2} \hat{\gamma}_{k, D}\right)}{\bar{\gamma}\left(1-\rho^{2}\right)}} \\
& \cdot I_{0}\left(\frac{2 \sqrt{\rho^{2} \gamma_{k, D} \hat{\gamma}_{k, D}}}{\bar{\gamma}\left(1-\rho^{2}\right)}\right)
\end{aligned}
$$

with $I_{0}(\cdot)$ standing for the zero-order modified Bessel function of the first kind.

\section{Outage Probability}

We define the outage probability as the probability that the instantaneous capacity of the system is below a predefined value $R$. Since we consider a two-hop DF scenario, we should start the analysis by studying the decoding subset $\mathcal{D S}$, i.e., the subset of relays able to decode the information transmitted by the source in the source-to-relay link:

$$
\mathcal{D S}=\left\{k: \log _{2}\left(1+\gamma_{S, k}\right) \geq 2 R\right\}=\left\{k: \gamma_{S, k} \geq 2^{2 R}-1\right\}
$$

Note that we have considered that relays are able to decode the source's information in the first hop when instantaneous capacity is higher than $2 R$, with this consideration also adopted in the relay-to-destination link. By doing so, the resulting end-to-end spectral efficiency is $R$ as the proposed twohop scheme requires two time-slots to transmit the information from the source to the destination.

For each combination of relays in the decoding set, the probability that the selected relay is not able to retransmit the source's information to the destination must be computed to obtain the outage probability of the system. By defining now $\mathcal{D S}_{p}^{l}$ as the $p$-th element of the set of all possible decoding subsets with $l$ relays (i.e., $\mathcal{D S}_{p}^{l}$ is the $p$-th decoding subset of the $\left(\begin{array}{c}K \\ l\end{array}\right)$ possible subsets of $l$ relays taken from the $K$ relays), we can easily compute the outage probability as [6]:

$P_{\text {out }} \triangleq \operatorname{Prob}($ outage $)=\sum_{l=0}^{K} \sum_{p=1}^{\left(\begin{array}{c}K \\ l\end{array}\right)} \operatorname{Prob}\left(\right.$ outage $\left.\mid \mathcal{D} \mathcal{S}_{p}^{l}\right) \operatorname{Prob}\left(\mathcal{D} \mathcal{S}_{p}^{l}\right)$

where $\operatorname{Prob}\left(\right.$ outage $\left.\mid \mathcal{D} \mathcal{S}_{p}^{l}\right)$ is the probability of outage conditioned on that the decoding subset is $\mathcal{D} \mathcal{S}_{p}^{l}, \operatorname{Prob}\left(\mathcal{D} \mathcal{S}_{p}^{l}\right)$ is the probability of that subset and $P_{\text {out }}$ has been defined for the sake of notation simplicity.

Now, the outage probability of the proposed scenario can be obtained according to the following Theorem (where parameter $y$ has been defined as $y=2^{2 R}-1$ and the dependance of $P_{\text {out }}$ on $y$ has been included for notation convenience).

Theorem 1: Consider a relay scenario as described in Section II where the actual channel state and its estimate can be related with parameter $\rho$. Then the outage probability of the system can be expressed as follows:

$$
\begin{aligned}
& P_{\text {out }}(y)=\left(1-e^{-\frac{y}{\gamma}}\right)^{K} \\
& +\sum_{l=1}^{K} l \sum_{m=0}^{l-1}\left(\begin{array}{c}
l-1 \\
m
\end{array}\right) \frac{(-1)^{m}}{m+1}\left(1-e^{-y \frac{m+1}{\bar{\gamma}\left(1+m\left(1-\rho^{2}\right)\right)}}\right) \\
& \cdot\left(\begin{array}{c}
K \\
l
\end{array}\right)\left(1-e^{-\frac{y}{\gamma}}\right)^{K-l} e^{-\frac{y l}{\gamma}}
\end{aligned}
$$


Proof: The proof is provided in the Appendix.

From the above expression, however, it is difficult to analyze the behavior of the system. In order to gain some insight, we consider the asymptotic high SNR regime in the next section.

\section{AsYmptotic AnALYsis}

Before starting to analyze the scheme with outdated CSI, it is worth noting that one can easily verify after some algebra that when $\rho=1$ (ideal scenario) the outage probability derived in the previous section simplifies to the well-known expression:

$$
P_{\text {out }}(y)=\left(1-e^{-\frac{2 y}{\gamma}}\right)^{K}
$$

which can be expressed as follows for the asymptotic SNR regime $(\bar{\gamma} \rightarrow \infty)$ :

$$
P_{\text {out }}(y)=\left(\frac{2 y}{\bar{\gamma}}\right)^{K}+o\left(\left(\frac{1}{\bar{\gamma}}\right)^{K}\right)
$$

By defining diversity order as $d=\lim _{\bar{\gamma} \rightarrow \infty}-\log \left(P_{\text {out }}\right) / \log (\bar{\gamma})$, one can easily check that the proposed scheme attains diversity order $K$ when the CSI knowledge is perfect. However, for $\rho<1$ the diversity of the system is substantially penalized as shown below.

Theorem 2: Let a scenario with asymptotic high SNR. Then, the outage expression can be expressed as:

$$
P_{\text {out }, \rho_{\text {high }}}(y)=K ! \frac{y}{\bar{\gamma}}\left(1-\rho^{2}\right)^{K-1}+o\left(\frac{1}{\bar{\gamma}}\right)
$$

for high values of $\rho\left(\rho \rightarrow 1^{-}\right)$, whereas for low values of $\rho$ $\left(\rho \rightarrow 0^{+}\right)$the outage approximation amounts to:

$$
P_{\text {out }, \rho_{\text {low }}}(y)=\frac{y}{\bar{\gamma}}\left(1+\rho^{2}\left(1-\sum_{k=1}^{K} 1 / k\right)\right)+o\left(\frac{1}{\bar{\gamma}}\right)
$$

Proof: The proof is provided in the Appendix.

It is worth noting that we have only analyzed the cases $\rho \approx 1$ and $\rho \approx 0$ for the ease of mathematical tractability. As we will show later, however, the study is very relevant as it shows that the loss of diversity gain is independent of the level of CSI accuracy. In the first case, we observe that outage probability decays exponentially as a function of the number of relays. In the case that $\rho \approx 0$, however, we observe that using more relays reduces the outage capacity following the well-known selection gain term $\sum_{k=1}^{K} 1 / k$. However, the sensibility of such reduction in terms of the number of relays is substantially lower than in the previous case. This effect comes from the fact that the selection tends to be random when $\rho$ is close to 0 and, then, selection gains cannot be efficiently exploited. As a final remark, it can be clearly observed that in both cases the diversity order is the same, as expressed by the following Corollary.

Corollary 1: The diversity order of the proposed relay selection mechanism can only take two possible values:

$$
d= \begin{cases}1 & \text { if } \rho<1 \\ K & \text { if } \rho=1\end{cases}
$$

Proof: For the cases $\rho=1, \rho=0, \rho \approx 1$ and $\rho \approx 0$, the corollary immediately results from applying the diversity definition, $d=\lim _{\bar{\gamma} \rightarrow \infty}-\log \left(P_{\text {out }}\right) / \log (\bar{\gamma})$, to (3)-(5). For the remaining values of $\rho$, the proof can also easily derived as follows. Clearly, the outage probability is a decreasing function in $\rho$. Therefore, by defining $\rho^{\prime}$ as an arbitrary value of $\rho$ (different from the cases $\rho=1, \rho=0, \rho \approx 1$ and $\rho \approx 0$ ), the following expression can be written:

$$
P_{\text {out }, \rho_{\text {high }}}(y) \leq P_{\text {out }, \rho^{\prime}}(y) \leq P_{\text {out }, \rho_{\text {low }}}(y)
$$

By applying now the diversity definition in the expression above, one can verify that the following inequalities hold:

$$
d_{\rho_{\text {low }}} \leq d_{\rho^{\prime}} \leq d_{\rho_{\text {high }}}
$$

Since the diversity order is the same for the extreme cases $\left(d_{\rho_{\text {low }}}=d_{\rho_{\text {high }}}=1\right)$, one can conclude that the diversity order is always equal to one when $\rho<1$.

Although the behavior of the diversity gain could seem surprising at first sight (specially for $\rho \approx 1$ ), the observed effect has a logical physical interpretation. When $\rho \neq 1$ there exists a non-zero probability that the worst relay is chosen. This relay contributes with a $1 / \bar{\gamma}$ term to the outage probability (i.e., diversity gain of order 1). As the SNR grows this effect is emphasized, becoming in the asymptotic regime the dominant term of the outage probability expression (as proven in the Appendix).

\section{Numerical Evaluation AND Discussion}

As far as the numerical evaluation is concerned, we consider a scenario with $K=5$ relays and target rate $R=1 \mathrm{bit} / \mathrm{s} / \mathrm{Hz}$. In Figure 2, we present outage probability results obtained for values of $\rho=1,0.995$ and 0.1 . To facilitate the comparison, we also provide results related to the case with a single relay $(K=1)$. As observed in the figure, the theoretical expression given by (2) completely agrees with the simulated curves. Concerning the derived high SNR approximations, these are quite accurate for SNRs beyond 15 and $35 \mathrm{~dB}$ for low and high $\rho$ values, respectively. As expected, better results are obtained by increasing $\rho$ but, even in the case $\rho=0.995$, the diversity gain is always one when $\rho \neq 1$. This can be clearly observed in the figure as the curves obtained for outdated CSI are parallel to the case with $K=1$. It is worth noting that an additional gain is obtained with $\rho=0.1$ when compared to the single relay case. Apart from the selection gain discussed in the previous section, this effect is motivated by the fact that the reliability of the source-to-relay link improves with $K$ as there exist more candidates for the decoding subset. Finally, one can observe a similar behavior for the slope of the curves associated with $\rho=0.995$ and $\rho=1$ in the medium SNR range. This is because the impact of a possible wrong relay selection on system performance is emphasized in the high-SNR region. Therefore, a deeper study in the medium SNR regime is necessary as practical systems usually work there. Nonetheless, this issue is out of the scope of this paper and is left for future research.

\section{APPENDIX}

In this appendix we provide the proofs of Theorems 1 and 2 . 


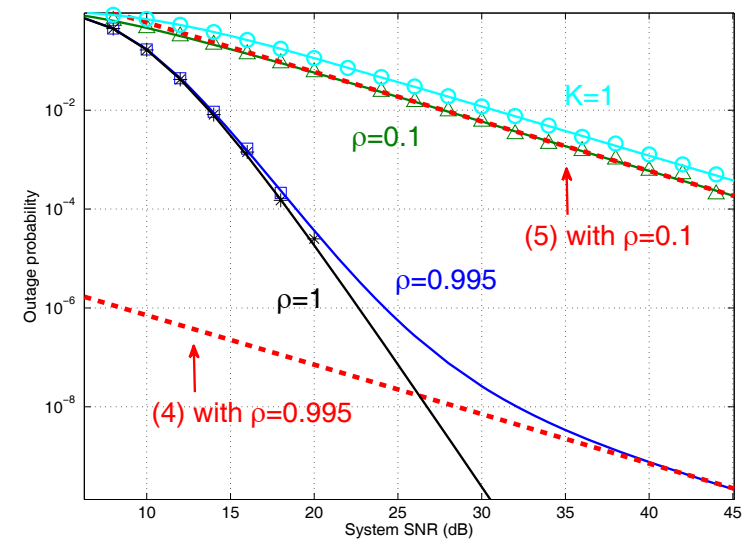

Fig. 2: Outage probability vs. system SNR, $P / \sigma_{n}^{2}$, for different values of $\rho(K=5$ relays, $R=1 \mathrm{bit} / \mathrm{s} / \mathrm{Hz}$. Symbol: simulation, solid curves: theoretical, dashed curves: high SNR approximation).

\section{A. Proof of Theorem 1}

We start the proof by noting that both the values of $\operatorname{Prob}\left(\mathcal{D S}_{p}^{l}\right)$ and $\operatorname{Prob}\left(\right.$ outage $\left.\mid \mathcal{D S}_{p}^{l}\right)$ in (1) are the same for a given value of $l$. In other words, these probabilities are independent of the combination of relays forming the decoding subset, $\mathcal{D S}$, when the number of active relays is fixed. The reason for that is that we assume an homogenous scenario where all the channels are i.i.d.. As a consequence, these probabilities only depend on the number of active relays (i.e., the cardinality of $\mathcal{D S}, \operatorname{card}(\mathcal{D S})$ ) and we can rewrite (1) as:

$$
P_{\text {out }}(y)=\sum_{l=0}^{K} \operatorname{Prob}(\text { outage } \mid \operatorname{card}(\mathcal{D S})=l) \operatorname{Prob}(\operatorname{card}(\mathcal{D S})=l)
$$

where $\operatorname{Prob}(\operatorname{card}(\mathcal{D S})=l)$ is the probability that the decoding subset has $l$ relays. By recalling the Rayleigh fading assumption, one can obtain this probability as follows:

$$
\operatorname{Prob}(\operatorname{card}(\mathcal{D S})=l)=\left(\begin{array}{c}
K \\
l
\end{array}\right)\left(1-e^{-\frac{y}{\gamma}}\right)^{K-l} e^{-\frac{y l}{\bar{\gamma}}}
$$

Notice that the above expression does not depend on the correlation parameter $\rho$. This is because a node belongs to the decoding set if it has perfectly decoded the information, which is independent of relay selection decisions.

Concerning Prob(outage $\mid \operatorname{card}(\mathcal{D S})=l)$, this is the probability that the selected relay (out of $l$ relays in $\mathcal{D S}$ ) is in outage. Clearly, this probability is equal to one when there are no relays to retransmit the information (i.e., $\operatorname{card}(\mathcal{D S})=0$ ). For $l>0$, Prob (outage $\mid \operatorname{card}(\mathcal{D S})=l$ ) can be obtained by noticing that this is a similar problem to that observed in a multiuser scenario where the user with the highest SNR is selected for transmission but available CSI is subject to delays [9]. With this in mind, we can use the cumulative density function (CDF) of the scheduled user's SNR obtained in [9, Eq. 8] to compute the probability that the selected relay is in outage (i.e., its SNR is lower than $y=2^{2 R}-1$ ):

$$
\begin{gathered}
\operatorname{Prob}(\text { outage } \mid \operatorname{card}(\mathcal{D S})=l)=l \sum_{m=0}^{l-1}\left(\begin{array}{c}
l-1 \\
m
\end{array}\right) \frac{(-1)^{m}}{m+1} \\
\cdot\left(1-e^{-y \frac{m+1}{\bar{\gamma}\left(1+m\left(1-\rho^{2}\right)\right)}}\right) \quad l>0
\end{gathered}
$$

Finally, by plugging (8) and (7) into (6) and noting that the first term is related to the case that $l=0$, one can easily verify that expression (2) holds.

\section{B. Proof of Theorem 2}

In the asymptotic high SNR regime, we have that $1 / \bar{\gamma} \rightarrow 0$. Then, we can use the Taylor series of the exponential function centered at 0 (i.e., $e^{x}=1+x+x^{2} / 2 !+x^{3} / 3 !+\ldots$ ) to rewrite (8) as:

$$
\begin{gathered}
\operatorname{Prob}(\text { outage } \mid \operatorname{card}(\mathcal{D S})=l)=l \sum_{m=0}^{l-1}\left(\begin{array}{c}
l-1 \\
m
\end{array}\right) \frac{(-1)^{m}}{m+1} \\
\cdot\left[\sum_{s=1}^{K} \frac{y^{s}(-1)^{s-1}}{s !} \mathcal{I}(s, l)+o\left(\left(\frac{1}{\bar{\gamma}}\right)^{K+1}\right)\right]
\end{gathered}
$$

where we have defined $\mathcal{I}(s, l)$ as follows:

$$
\mathcal{I}(s, l)=\left(\frac{1}{\bar{\gamma}}\right) \sum_{m=0}^{s}\left(\begin{array}{c}
l-1 \\
m
\end{array}\right) \frac{(-1)^{m}}{m+1}\left(\frac{m+1}{\left(1+m\left(1-\rho^{2}\right)\right)}\right)^{s}
$$

From the above expressions, one can observe that the problem is reduced to compute $\mathcal{I}(s, l)$. According with the value of $\rho$ the behavior of such an expression differs and, for that reason, we have divided the proof in two parts:

- High $\rho$ region $\left(\rho \rightarrow 1^{-}\right)$:

In this case, $1-\rho^{2} \rightarrow 0$ and we can resort to the Taylor series centered at $x=0$ of $1 /(1+m x)^{s}$ :

$$
\begin{aligned}
\frac{1}{(1+m x)^{s}}=1 & -s m x \\
& +\frac{s(s+1) m^{2} x^{2}}{2 !}-\frac{s(s+1)(s+2) m^{3} x^{3}}{3 !}+\ldots
\end{aligned}
$$

to rewrite (10) as follows $\left(x=1-\rho^{2}\right)$ :

$$
\begin{aligned}
\mathcal{I}(s, l)= & \left(\frac{1}{\bar{\gamma}}\right) \sum_{m=0}^{s}\left(\begin{array}{c}
l-1 \\
m
\end{array}\right)(-1)^{m}(m+1)^{s-1} \\
& \cdot\left(1-s m\left(1-\rho^{2}\right)+\frac{s(s+1) m^{2}\left(1-\rho^{2}\right)^{2}}{2 !}\right. \\
& \left.-\frac{s(s+1)(s+2) m^{3}\left(1-\rho^{2}\right)^{3}}{3 !}+\ldots\right)
\end{aligned}
$$

Notice that due to the asymptotic high SNR regime assumption $(1 / \bar{\gamma} \rightarrow 0)$, the dominant terms in (9) are those depending on $\mathcal{I}(s=1, l)$. Therefore, we can focus our attention on obtaining the value of $\mathcal{I}(1, l)$ which can be computed with the help of the following equalities [10]:

$$
\begin{array}{lr}
\sum_{k=0}^{N}(-1)^{k}\left(\begin{array}{l}
N \\
k
\end{array}\right) k^{n-1}=0 & 1 \leq n \leq N \\
\sum_{k=0}^{n}(-1)^{k}\left(\begin{array}{l}
n \\
k
\end{array}\right) k^{n}=(-1)^{n} n ! & n \geq 0
\end{array}
$$


as follows:

$$
\mathcal{I}(1, l)=\left(\frac{1}{\bar{\gamma}}\right)\left[(l-1) !\left(1-\rho^{2}\right)^{l-1}+o\left(\left(1-\rho^{2}\right)^{l-1}\right)\right]
$$

By plugging the above equation in (9), we have that:

$$
\begin{aligned}
\operatorname{Prob}(\text { outage } \mid \operatorname{card}(\mathcal{D S})=l)= & \left(\frac{y}{\bar{\gamma}}\right) l !\left(1-\rho^{2}\right)^{l-1} \\
& +o\left(\left(1-\rho^{2}\right)^{l-1}\right) o\left(\frac{1}{\bar{\gamma}}\right)
\end{aligned}
$$

Now, by substituting the above expression in (2) and by also considering the Taylor series centered at 0 of the terms coming from (7) (i.e., $\operatorname{Prob}(\operatorname{card}(\mathcal{D S})=l)=\left(\begin{array}{c}K \\ l\end{array}\right)(y / \bar{\gamma})^{K-l}(1-$ $\left.y / \bar{\gamma})^{l}+o\left((1 / \bar{\gamma})^{K-l}\right)\right)$, we can obtain the following result:

$$
\begin{aligned}
P_{\text {out }}(y)= & \left(\frac{y}{\bar{\gamma}}\right)^{K}+o\left(\left(\frac{1}{\bar{\gamma}}\right)^{K}\right) \\
& +\sum_{l=1}^{K}\left[\left(\left(\frac{y}{\bar{\gamma}}\right) l !\left(1-\rho^{2}\right)^{l-1}+o\left(\frac{\left(1-\rho^{2}\right)^{l-1}}{\bar{\gamma}}\right)\right)\right. \\
& \left.\cdot\left(\left(\begin{array}{c}
K \\
l
\end{array}\right)\left(\frac{y}{\bar{\gamma}}\right)^{K-l}\left(1-\frac{y}{\bar{\gamma}}\right)^{l}+o\left(\left(\frac{1}{\bar{\gamma}}\right)^{K-l}\right)\right)\right] \\
& =\left(\frac{y}{\bar{\gamma}}\right)^{K}+\sum_{l=1}^{K}\left(\begin{array}{c}
K \\
l
\end{array}\right)\left(\frac{y}{\bar{\gamma}}\right)^{K-l+1} \\
& \cdot l !\left(1-\rho^{2}\right)^{l-1}\left(1-\frac{y}{\bar{\gamma}}\right)^{l}+o\left(\frac{1}{\bar{\gamma}}\right)
\end{aligned}
$$

We can observe in (13) that the predominant term is that depending on $1 / \gamma$ (i.e., the term $l=K$ in the summation). By focusing on this term and by considering that $1-\frac{y}{\bar{\gamma}} \approx 1$, result given by (4) is then obtained.

Before introducing the case $\rho \approx 0$, let us introduce some remarks on the obtained approximation. Notice that for $K=1$, the summation in (13) has two terms and both of them depends on $1 / \bar{\gamma}$. As a consequence, none of these two terms can be neglected, thus obtaining the expression given by (3). By using the derived approximation in (4), the approximation would not be valid as a $3 \mathrm{~dB}$ gain would be observed. On the other hand, in the case that $\rho$ is exactly equal to 1 , the approximated result given by (4) is equal to 0 , which is not true. The problem with that expression resides in the fact that equation (11) for $\rho=1$ would be reduced to:

$$
\mathcal{I}(s, l)=\left(\frac{1}{\bar{\gamma}}\right)^{s} \sum_{m=0}^{l-1}\left(\begin{array}{c}
l-1 \\
m
\end{array}\right)(-1)^{m}(m+1)^{s-1}
$$

After some algebra and with the help of expressions in (12), one can easily check that $\mathcal{I}(1, l)=\mathcal{I}(2, l)=\ldots=\mathcal{I}(l-1, l)=0$ and only terms $s \geq l$ are different from 0 . Hence, one can then prove that $\operatorname{Prob}($ outage $\mid \operatorname{card}(\mathcal{D S})=l)=(y / \bar{\gamma})^{l}+o\left((y / \bar{\gamma})^{l}\right)$ (details are omitted here for brevity). By using this new expression in (13), one should observe that all the terms depend on $1 / \gamma^{K}$. As a consequence, there are no dominant terms in the summation, being the resulting approximation equal to (3)). It is worth noting, however, that these are the only problems appearing with the proposed approximation.
This last affirmation is supported by the numerical results provided in Section V.

- Low $\rho$ region $\left(\rho \rightarrow 0^{+}\right)$:

The procedure developed in this case is quite similar to the previous one. The main difference is that (since $1-\rho^{2} \approx 1$ ) Taylor series of $1 /(1+m x)^{s}$ in (10) is now developed centered at $x=1$. By doing so, we can express $\mathcal{I}(1, l)$ as:

$$
\begin{aligned}
\mathcal{I}(1, l)= & \left(\frac{1}{\bar{\gamma}}\right) \sum_{m=0}^{l-1}\left(\begin{array}{c}
l-1 \\
m
\end{array}\right)(-1)^{m} \\
& \cdot\left(\frac{1}{1+m}+\frac{m \rho^{2}}{(1+m)^{2}}+o\left(\rho^{2}\right)\right)
\end{aligned}
$$

After some manipulations and with the help of [10, Eq. 0.155.4] one can verify that the following result holds:

$$
\begin{aligned}
\operatorname{Prob}(\text { outage } \mid \operatorname{card}(\mathcal{D S})=l)= & \frac{y}{\bar{\gamma}}\left(1+\rho^{2}\left(1-\sum_{m=1}^{l} 1 / m\right)\right) \\
& +o\left(\rho^{2}\right) o\left(\frac{1}{\bar{\gamma}}\right)
\end{aligned}
$$

Now, by following the same approach as carried out to obtain (13), one would observe that the term $l=K$ is again the dominant term. As a result, one can readily verify that (5) holds. Notice, however, that the obtained approximation would fail for $K=1$. This is motivated again by the fact that some terms cannot be neglected when $K=1$ and, then, the reasoning discussed for the previous case $\rho \approx 1$ is also valid here.

\section{ACKNOWLEDGMENT}

The authors would like to thank the anonymous reviewers for their comments on the paper.

\section{REFERENCES}

[1] A.Sendonaris, E. Erkip, and B. Aazhang, "User cooperation diversity, part I and II," IEEE Trans. Commun., vol. 51, no. 11, Nov. 2003.

[2] J. N. Laneman, D. Tse, and G. W. Wornell, "Cooperative diversity in wireless networks: efficient protocols and outage behaviour," IEEE Trans. Inform. Theory, vol. 50, no. 12, pp. 3062-3080, Dec. 2004.

[3] R. U. Nabar, H. Bolcskei, and F. W. Kneubuler, "Fading relay channels: performance limits and space-time signal design," IEEE J. Select. Areas Commun., Aug. 2004.

[4] D. Gesbert, M. Shafi, D. Shiu, and P. Smith, "From theory to practice: an overview of space-time coded MIMO wireless systems," IEEE $J$. Select. Areas Commun., Apr. 2003.

[5] A. Bletsas, A. Khisti, D. P. Reed, and A. Lippman, "A simple cooperative diversity method based on network path selection," IEEE J. Select. Areas Commun., vol. 24, no. 3, pp. 659-672, Mar. 2006.

[6] A. Bletsas, H. Shin, and M. Z. Win, "Cooperative communications with outage-optimal opportunistic relaying," IEEE Trans. Wireless Commun., vol. 6, no. 9, Sept. 2007.

[7] B. Zhao and M. C. Valent, "Practical relay networks: a generalization of hybrid-ARQ," IEEE J. Select. Areas Commun., vol. 23, no. 1, pp. 7-18, Jan. 2005.

[8] A. Bel, J. Vicario, and G. Seco-Granados, "The benefits of relay selection in WiMAX networks," in Proc. ICT Mobile Summit, 2008.

[9] J. Vicario and C. Antón-Haro, "Analytical assessment of multi-user vs. spatial diversity trade-offs with delayed channel state information," IEEE Commun. Lett., Aug. 2006.

[10] I. Gradsthteyn and I. Ryzhik, Tables of Integrals; Series and Products. New York: Academic, 1965. 